\title{
Technique of immediate reconstruction with tissue expanders after mastectomy for breast cancer
}

\author{
Michael Friedrich* and Stefan Krämer \\ Department of Obstetrics and Gynaecology with Breast Cancer Center, HELIOS-Hospital, Lutherplatz 40, 47805 Krefeld, Germany
}

\begin{abstract}
Tissue expanders are regarded as a simple method for immediate breast reconstruction following mastectomy. However, to achieve a satisfying cosmetic result and avoid complications associated with the procedure several technical aspects and a careful selection of patients is required. Early ccomplications of tissue expansion are skin necrosis with wound dehiscence and implant extrusion. In case the viability of the skin flaps is in doubt the expansion process should be delayed and any nonviable tissue should be excised early to allow secondary wound healing. The expansion is started no earlier than wound healing is completed, and viability of mastectomy flaps is secured. Complete muscular coverage of the expander reduces the risk for expander extrusion in case of wound infection or wound dehiscence. In case of ptosis of the contralateral breast overexpansion is needed to get an acceptable ptosis. If necessary, the mobilisation of the lower part can be extended downwards to the rectus sheath to gain an excess amount of skin which is used to create a submammary fold. When the expansion is finished the excess amount of skin is fixed to the muscle fascia. Another possibility is to overexpand and exchange the expander to a slightly smaller implant. It is mandatory to leave the suction drains until drainage is less than $20 \mathrm{cc}$ for 2 consecutive days. This avoids seromas which are related to a higher risk for capsular fibrosis.
\end{abstract}

\section{Concept}

Tissue expanders are regarded as a simple method for immediate breast reconstruction following mastectomy $[1,2]$. However, to achieve a satisfying cosmetic result and avoid complications associated with the procedure several technical aspects and a careful selection of patients is required [3-6].

After the completion of mastectomy a tissue expander is inserted under a muscular pocket and then inflated to expand the dermomuscular layer. When the expansion is finished the device is changed to a permanent implant.

Today textured surface anatomical breast-shaped expanders are used or permanent expander implants (adjustable saline filled inner volume, silicone gel outer volume), which avoid the need of an exchange of the expander to a permanent implant [7-9].

The aims of reconstruction with expanders are to restore the breast shape and volume as close as possible to the contralateral breast and to reconstruct the inframammary fold with adequate ptosis.

\section{Indication}

Immediate reconstruction with expanders is recommended in patients:

- with small or moderate-sized breast with minimal or no ptosis

- who want minimal scarring and no additional donor-site morbidity

- with no prior radiation / no postoperative radiation planned

- are not worried about a silicone implant

- are unwilling or unfit to undergo autologous tissue reconstruction.

- undergoing bilateral reconstruction (and meet the criteria mentioned above)
- In most patients a contralateral mastopexy / reduction is required for symmetry.

\section{Contraindication}

- poor quality of the soft tissue coverage (skin and muscle) does not allow expansion

- large / ptotic breasts

- prior radiation or radiation planned after surgery

- obese patients / patients with large chest wall diameters

- low patient compliance for the expansion process

- patients with unrealistic cosmetic expectations

young patients (relative contraindication for more re-operations may be required during life-span)

\section{Preoperative drawings}

The type mastectomy and the amount of skin to be excised should be determined prior to surgery. Skin-sparing techniques are preferred for they leave a skin envelope which aids in reconstruction. Skin very close to or infiltrated by the tumor is excised, narrow skin bridges should be avoided to ensure adequate blood supply of the skin flaps.

The central midline, the vertical breast axis and both inframammary folds are outlined with an additional line on the mastectomy side $1 \mathrm{~cm}$

${ }^{*}$ Correspondence to: Michael Friedrich, Department of Obstetrics and Gynaecology with Breast Cancer Center, HELIOS-Klinikum, Lutherplatz 40, 47805 Krefeld, Germany, E-mail: michael.friedrich@helios-gesundheit.de

Received: September 14, 2018; Accepted: September 21, 2018; Published: September 24, 2018 
lower than the existing fold. The shape and contour of the new breast is outlined in accordance to the contralateral breast (Figure 1). Base width and height of the contralateral breast are measured and transferred to the tumor side (the markings may also be drawn using manufacturer's templates).

\section{Surgery}

After completion of the (skin-sparing) mastectomy the viability of the skin flaps and the integrity of the pectoralis major muscle are assessed (Figure 2a). Nonviable skin is excised and lesions in the muscle are fixed before insertion of the expander.

\section{Insertion of the expander}

The lateral border of the pectoralis major muscle is incised (Figure $2 \mathrm{~b})$ and a submuscular pocket is created with the pectoralis major muscle released medially from the third intercostal space down and inferiorly (Figure 2c). The inferior part of the dissection is either subcutaneously or includes the anterior rectus sheath which is then elevated in continuity with the pectoralis major muscle. When total muscular coverage is planned the serratus anterior muscle is elevated from the chest wall to provide lateral coverage (Figure 2d,e).

The size and type of expander used depend on the width and height of the contralateral breast and the volume may be estimated by the weight of the mastectomy specimen. The size of the expander is smaller

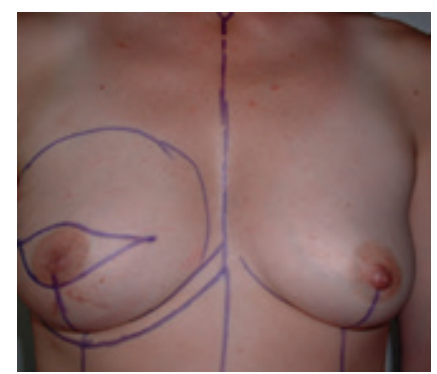

Figure 1. Preoperative drawings

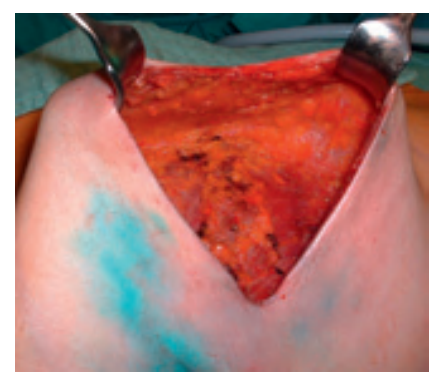

Figure 2a. Following mastectomy the viability of the skin flaps and the integrity of the pectoralis major muscle are evaluated

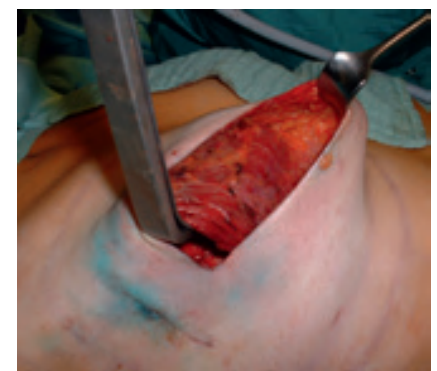

Figure 2b. The pectoralis major muscle is incised laterally

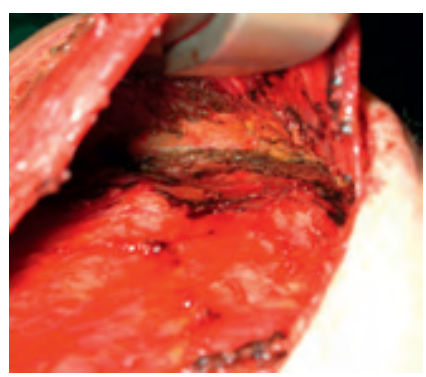

Figure 2c. A subpectoral pocket is prepared. The muscle is dissected medially and in the inframammary fold

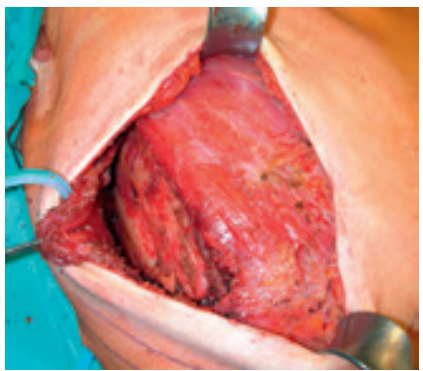

Figure 2d. The serratus muscle is dissected from the thoracic wall

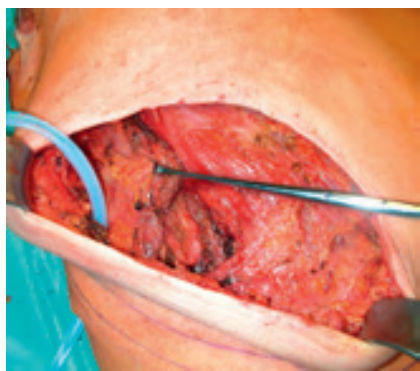

Figure 2e. The serratus muscle is mobilized that it can be sutured to the pectoralis major muscle to get a total submuscular pocket

when a contralateral breast reduction is planned or larger in case of a large or ptotic breast.

The expander is completely evacuated of air using a butterfly needle (Figure 2f). It is then partially inflated with sterile saline (may contain methylene blue to assure puncturing the expansion chamber during the expansion process) to assure that there is no leakage. About $50 \mathrm{cc}$ of saline are left within the expander and this aids in implant insertion. The prosthesis is brought in the submuscular pocket and oriented by help of markers on the implant surface (Figure $2 \mathrm{~g}$ ).

Drains are placed in the submuscular pocket and subcutaneously. The submuscular pocket is closed suturing the serratus and pectoralis muscle with interrupted Vicryl 3-0 sutures, which are pre-inserted before placement of the prosthesis to minimize risk of perforation of the implant by the needle (Figure $2 \mathrm{~h}$ ).

Interrupted sutures Vicryl 4-0 can be used for adapting the subcutaneous tissues and monocryl $4-0$ for non-interrupted intracutaneous sutures. A bandage may be used for 3 weeks to keep the expander in place.

\section{Expander inflation}

The expander may be inflated immediately with saline $(100-300 \mathrm{cc})$ depending on the quality of the soft tissue coverage. Expansion begins 2-3 weeks following surgery but depends on the skin flap viability and 


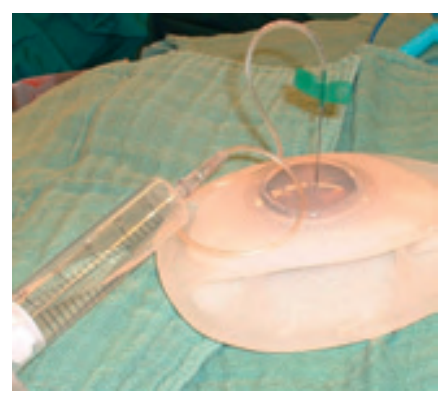

Figure 2f. The expander is completely evacuated of air using a butterfly needle

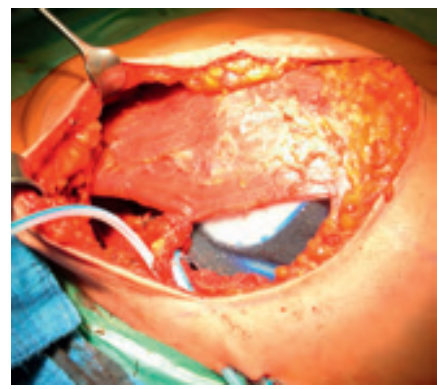

Figure 2g. The expander is in the submuscular position

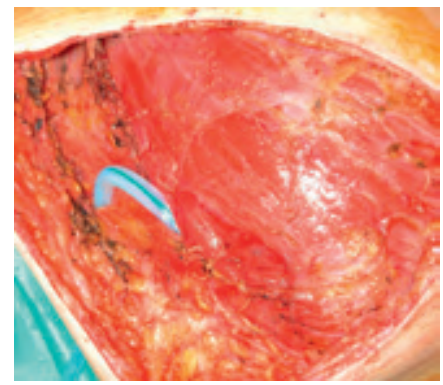

Figure 2h. The submuscular pocket is closed

wound healing. The expander is gradually expanded using magnetic port locators with $50-100 \mathrm{cc}$ of saline every 2-3 weeks until the desired volume is reached (Figure 3a-c). Usually the expander is slightly overexpanded to gain more tissue for creating a more natural ptosis.

\section{Expander to implant exchange}

Expansion is maintained 2-6 months. In case a permanent expander implant has been used the volume is adjusted according to the contralateral breast by aspirating saline. The fill tube is removed later under local anaesthesia.

In case of a temporary expander the patient is put in a sitting position and saline is aspirated from the expander until symmetry to the contralateral breast is reached (Figure 4a). The expander is removed through the previous incision (Figure $4 \mathrm{~b}$ ) and a capsulotomie (either circumferential or only in the inferior pole, with or without radial incisions) is done to release tension and enlarge the pocket (Figure 4c). The volume of the permanent implant is chosen according to the expander volume after symmetry has been reached or may be tested with sizers. Whether to choose an anatomic or a round shaped implant depends on the shape and the upper pole fullness of the contralateral breast (Figure 4d).

A drain is placed into the pocket and the incisions are closed. Final symmetry is evaluated in a sitting position (Figure 4e,f). A bandage may be used for 3 weeks to avoid cranial displacement of the implant.
The reconstruction of the nipple-areola complex is performed $3-6$ months later.

\section{Tips and tricks}

- Early ccomplications of tissue expansion are skin necrosis with wound dehiscence and implant extrusion. In case the viability of the skin flaps is in doubt the expansion process should be delayed and any nonviable tissue should be excised early to allow secondary wound healing. The expansion is started no earlier than wound healing is completed and viability of mastectomy flaps is secured

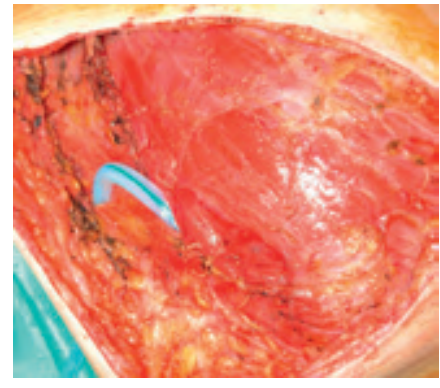

Figure 2h. The submuscular pocket is closed

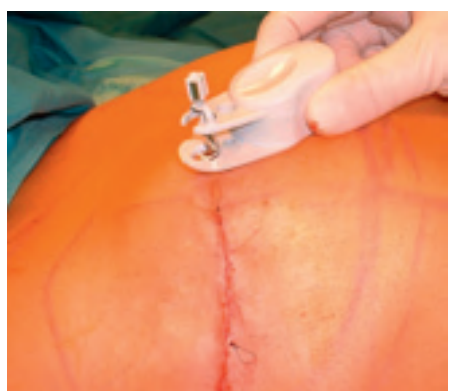

Figure 3a. With the help of a magnetic port locator the integrated valve is located for safe filling of the expander

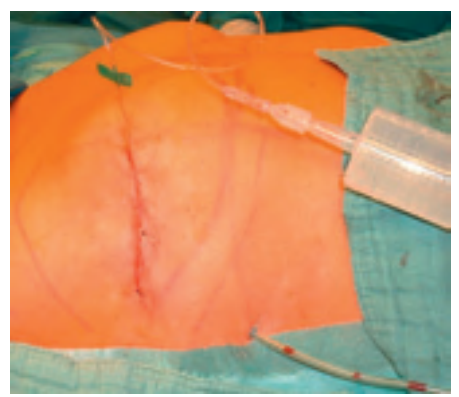

Figure 3b. The expander is filled with saline

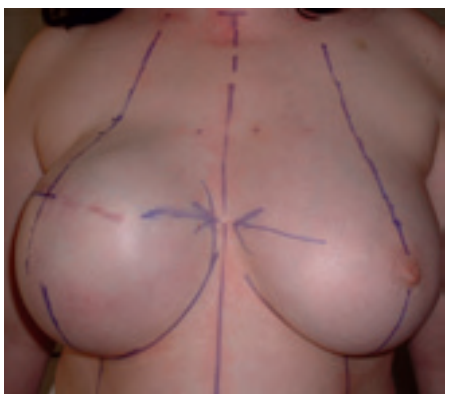

Figure 3c. The expander at the end of the expansion period (slightly overexpanded) 


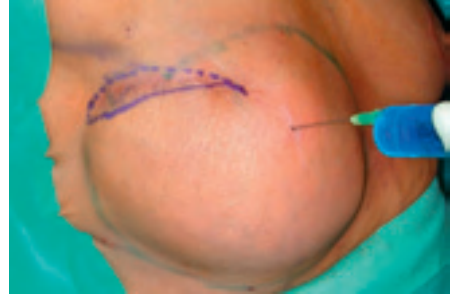

Figure 4a. The patient is in a sitting position and saline is aspirated from the expander until symmetry to the contralateral breast is reached

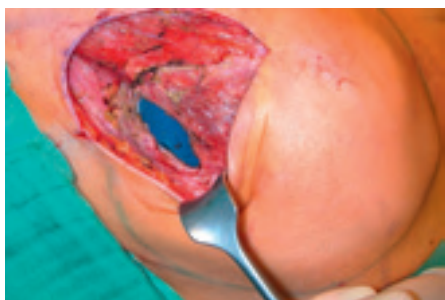

Figure 4b. The expander is removed through the previous incision

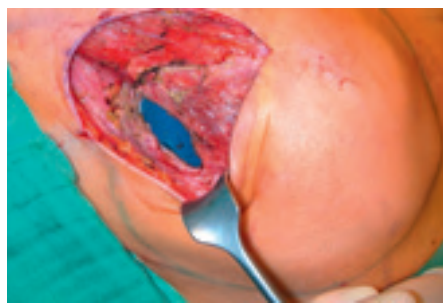

Figure 4c. A capsulotomie is done to release tension and enlarge the pocket

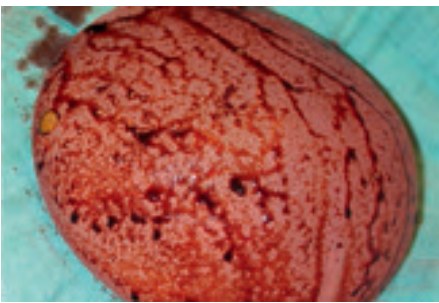

Figure 4d. For the permanent implant an anatomical shaped implant has been selected and the implant is rinsed in betadine solution

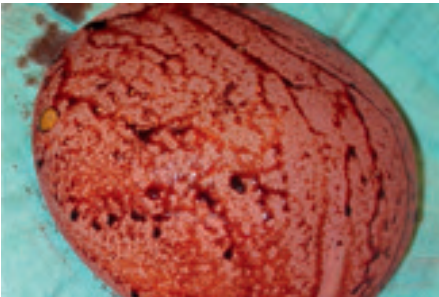

Figure 4d. For the permanent implant an anatomical shaped implant has been selected and the implant is rinsed in betadine solution

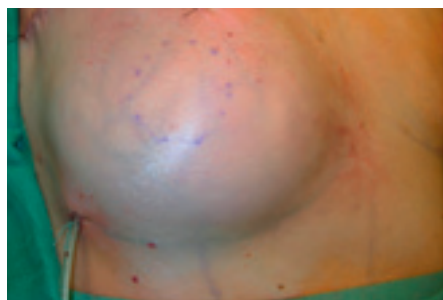

Figure 4e. Final result following insertion of the permanent implant

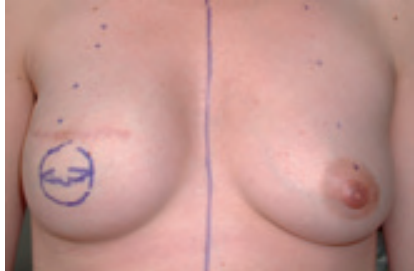

Figure 4f. Late postoperative view. Reconstruction of the nipple-areola complex is done $3-6$ months after exchange of the implant

- Complete muscular coverage of the expander reduces the risk for expander extrusion in case of wound infection or wound dehiscence

- In case of ptosis of the contralateral breast overexpansion is needed to get an acceptable ptosis. If necessary the mobilisation of the lower part can be extended downwards to the rectus sheath to gain an excess amount of skin which is used to create a submammary fold. When the expansion is finished the excess amount of skin is fixed to the muscle fascia. Another possibility is to overexpand and exchange the expander to a slightly smaller implant.

- In large or ptotic breasts a skin-reducing mastectomy or a mastectomy by a vertical elliptical incision is done to reduce the amount of skin

- Leave the suction drains until drainage is less than $20 \mathrm{cc}$ for 2 consecutive days. This avoids seromas which are related to a higher risk for capsular fibrosis.

- Most women require a contralateral mastopexy / reduction for symmetrization

- Concomitant chemotherapy may negatively influence the expansions process.

\section{References}

1. Maxwell GP, Falcone PA (1992) Eighty-four consecutive breast reconstructions using a textured silicone tissue expander. Plast Reconstr Surg 89: 1022-1034. [Crossref]

2. Cordeiro PG, McCarthy CM (2006) A single surgeon`s 12 year experience with tissue expander / implant breast reconstruction: Part I. A prospective analysis of early complications. Plast Reconstr Surg 118: 825-831. [Crossref]

3. Woods JE, Managan MA (1992) Breast reconstruction with tissue expanders: obtaining an optimal result. Ann Plast Surg 28: 390-396.

4. Friedrich M, Kolberg HC, Diedrich K, Krämer S (2005) Primäre und sekundäre alloplastische Brustrekonstruktionen. Gynäkologe 38: 209-215.

5. Friedrich M, Salehin D, Krämer S (2010) Immediate Reconstruction After Mastectomy: Reconstruction With Tissue Expanders. In: Fitzal F and Schrenk P (eds), Oncoplastic Breast Surgery - a Guide to Clinical Practice, pp 162 -168.

6. Spear SL, Newman MK, Bedford MS, Schwartz KA, Cohen M, et al. (2008) A retrospective analysis of outcomes using three common methods for immediate breast reconstruction. Plast Reconstr Surg 122: 340-347. [Crossref]

7. Spear SL, Majidian A (1998) Immediate breast reconstruction in two stages using textured, integrated-valve tissue expanders and breast implants: a retrospective review of 171 consecutive breast reconstructions from 1989 to 1996. Plast Reconstr Surg 101: 53-63. [Crossref]

8. Jones G (2005) Decision-making: sizing and system selection. In: Jackson IT (ed.) Innovations in Plastic Surgery: Tissue expansion in breast surgery Quality Medical Publishing, Inc. St. Louis, pp 27-38.

9. Becker H (2006) One-stage immediate breast reconstruction with adjustable implants. In: Spear SL (ed) Surgery of the breast: Principles and art. $1^{\text {st }}$ Edition. Philadelphia: Lippincot-Raven, 438-450.

Copyright: (C2018 Friedrich M. This is an open-access article distributed under the terms of the Creative Commons Attribution License, which permits unrestricted use, distribution, and reproduction in any medium, provided the original author and source are credited. 